8th Alexander Friedmann International Seminar

on Gravitation and Cosmology

International Journal of Modern Physics: Conference Series

Vol. 3 (2011) 475-481

(C) World Scientific Publishing Company

DOI: $10.1142 /$ S2010194511000997

\title{
GRAVITATIONAL LENSING OF COSMOLOGICAL NEUTRINO SOURCES
}

\author{
GUSTAVO E. ROMERO \\ Instituto Argentino de Radioastronomía, C.C.5, (1894) Villa Elisa, Bs. As., Argentina \\ romero@iar-conicet.gov.ar
}

Facultad de Ciencias Astronómicas y Geofísicas, Universidad Nacional de La Plata, Paseo del Bosque s/n, 1900, La Plata, Argentina

romero@fcaglp.unlp.edu.ar

FLORENCIA L. VIEYRO

Instituto Argentino de Radioastronomía, C.C.5, (1894) Villa Elisa, Bs. As., Argentina fvieyro@iar-conicet.gov.ar

Received 9 June 2011

\begin{abstract}
Long gamma-ray bursts are likely the result of the collapse of very massive stars into black holes. These events are expected to produce high-energy neutrinos through photomeson production. Such neutrinos can escape from the source and travel up to the Earth. In the case of Population III progenitors for gamma-ray bursts, the neutrinos can be the only source of information of the first stars formed in the universe. The expected signal is rather weak, but we propose that gravitational lensing by nearby supermassive black holes can offer a natural tool to enhance and give a chance to detect neutrinos from the re-ionization era of the universe with current instrumentation.
\end{abstract}

Keywords: Neutrino sources; $\gamma$-ray bursts; gravitational lensing.

PACS numbers: 95.85.Ry, 98.70.Rz, 95.30.Sf

\section{Introduction}

Gamma-ray bursts (GRBs) are the most violent and energetic explosions in the universe. There flux on the Earth can surpass all the remaining gamma-ray flux from the sky. These extraordinary events occur around once per day. Their completely isotropic distribution and the measurement of lines with large redshifts (up to 6.7) indicate an extragalactic origin. For reviews, see those of Piran, ${ }^{1}$ Topinka, ${ }^{2}$ and references therein.

GRBs come in two flavors: short $(\sim 0.1 \mathrm{~s})$ and long $(\sim 10 \mathrm{~s}$ or more). They are thought to be events related to the end of the life of some stars. In the case of short GRBs, they seem to be the result of the final merger of binary systems formed by two neutron stars or a neutron star and a black hole. Long GRBs, on the contrary, 
are likely associated with the final collapse of very massive magnetized stars to black holes. The transient object that exists during the collapse, formed by a black hole at the core of the star, a hyperdense accretion disk, a couple of ultra-relativistic jets, and the stellar envelope, is usually called a collapsar. ${ }^{3}$

The relativistic jets of collapsars can reach bulk Lorentz factors of more than 100. When they escape form the star, strong shocks are expected to convert a fraction of the kinetic bulk energy into energy of relativistic protons and electrons. Photomeson production then can lead to neutrino emission. ${ }^{4,5}$ The neutrino signal, however, is likely to be rather weak in many cases, making the detection with current instruments difficult. In this paper we propose that such a signal can be magnified by gravitational lensing produced by interposed supermassive black holes. In the case of Population III GRBs, such neutrinos can be of extraordinary cosmological importance, since they can be used to probe the universe at the re-ionization era.

\section{Collapsars}

The collapse of a very massive star is likely to occur through the formation of a black hole in its core. ${ }^{3}$ The accretion of the matter onto this black hole can take place at very high rates of $\sim 1 M_{\odot} \mathrm{yr}^{-1}$ or more. Since the matter has high angular momentum it would form an accretion disk of high density (close to nuclear density) in the stellar interior. The magnetic field is accreted along the inflow, twisted in the ergosphere of the black hole, and piled up generating a region of very high magnetic density. Magnetic pressure gradients propel charged particles along the rotation axis of the system. The result is the formation of jets that accelerate since they are strongly confined by the stellar layers. As the jet accelerates, magnetic energy is converted into kinetic energy. By the time the jet erupts from the stellar surface, it has an ultrarelativistic speed (Lorentz factors above 100 and magnetization parameter $^{\mathrm{a}}$ well below 1). The formation and acceleration of GRBs jets have been studied by several authors through extensive numerical simulations ${ }^{6-8}$ The duration of the GRB depends on the mass of the black hole, the accretion rate, and the initial mass of the progenitor star. For very massive type II stars with $M \sim 100 M_{\odot}$, the event can last $\sim 10 \mathrm{~s}$. In the case of stars of first generation, the so-called Population III stars, whose masses can reach $\sim 10^{3-4} M_{\odot}$, durations are up to $10^{3} \mathrm{~s}$ at energies $E>1 \mathrm{MeV}$. These stars would be at very high redshifts; hence most of the photons should be absorbed along their way through the universe. Neutrinos, however, can reach the Earth, carrying information of the first structures formed in the universe. In what follows we discuss briefly how neutrinos can be generated in collapsars.

\section{Neutrino Production in Collapsars}

The jet starts as a magnetic dominated and collimated outflow but the energy density in the field quickly decays. Shocks can develop only when the kinetic energy

a The magnetization parameter is defined as $\sigma=$ magnetic energy density/kinetic energy density. 
is larger than the magnetic one and the fluid is compressible, i.e. when $\sigma<<1$. This condition will be fulfilled by the time the jet erupts from the star. Then strong shocks are formed: a downward shock moving in the relativistic jet and the forward shock moving in the external medium. ${ }^{9}$ These shocks are expected to accelerate particles up to relativistic energies. ${ }^{10}$ The magnetic field in the jet should drop as $B(z)=B_{0}\left(z / z_{0}\right)^{-q}$, with $1<q<2, B_{0} \sim 10^{15} \mathrm{G}$, and $z_{0}$ the injection point over the black hole ( $\sim$ a few tens of gravitational radii). At the surface of the star $\left(z=R_{\star} \sim 10^{12} \mathrm{~cm}\right)$ the field can still be as high $10^{6} \mathrm{G}$. This means that the reverse shock will produce particles that will cool quickly by synchrotron radiation. In Figs. 1 and 2 we show the radiative losses and decay timescales for charged pions and muons in a magnetized plasma with magnetic field of $6 \times 10^{5}$ G. ${ }^{11}$ From these plots it is clear that all muons with energies above $E_{\mu} \sim 10^{13} \mathrm{eV}$ are cooled with the consequent neutrino attenuation. For pions, suppression of high energy neutrinos occur above $\sim 10^{14} \mathrm{eV}$. As pointed out by some authors, ${ }^{12}$ these effects lead to a significant neutrino signal attenuation in instruments like to IceCube, sensitive only to high energy neutrinos $\left(E_{\nu}>1 \mathrm{TeV}\right)$.

The production of neutrinos can be more efficient in the shocked region of the ambient medium whereas the forward shock propagates. ${ }^{10}$ There, the magnetic field should be much lower, and synchrotron losses for secondaries might be negligible. Studies of neutrino production in Population III gamma-ray bursts suggest a total number of muon neutrinos in IceCube of $\sim 1-4$ depending on the black hole mass and other model details. ${ }^{10}$ If the external magnetic field is dominated by the field of the star, which could be as high as $10^{3} \mathrm{G}$, then the neutrino flux from

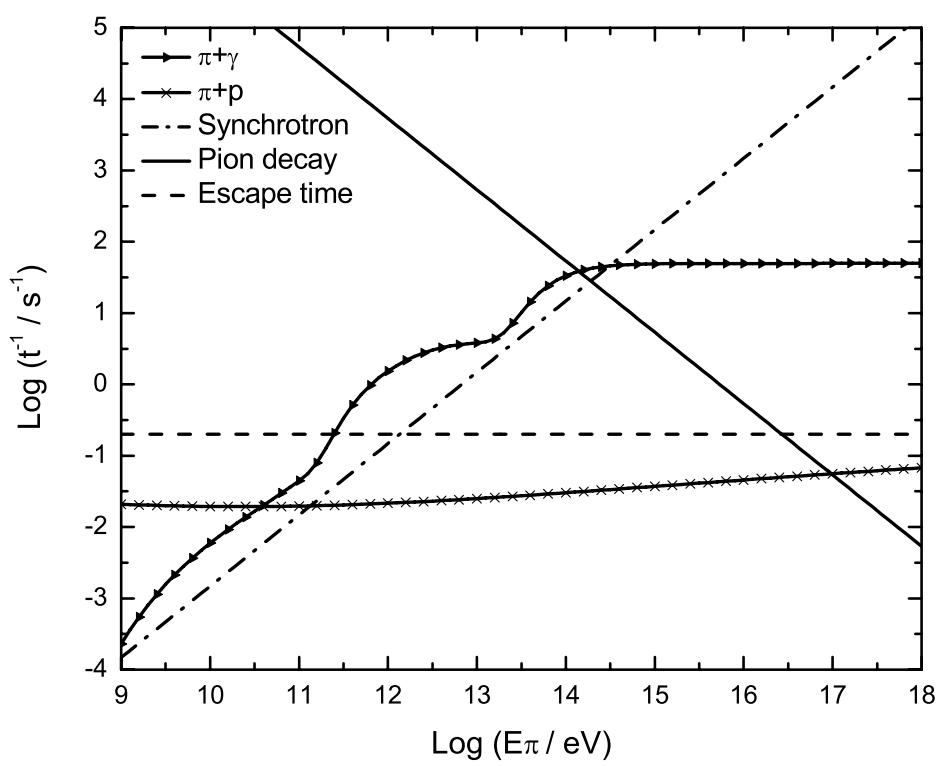

Fig. 1. Energy losses for charged pions in a magnetized plasma $\left(B=6 \times 10^{5} \mathrm{G}\right)$. 


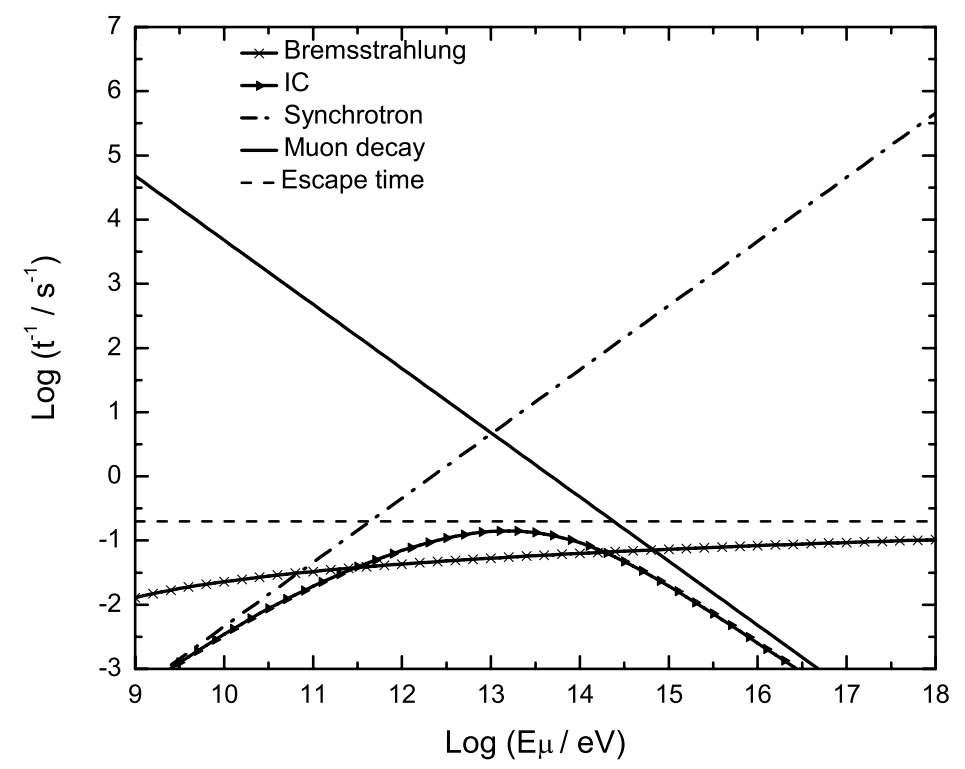

Fig. 2. Energy losses for muons in a magnetized plasma $\left(B=6 \times 10^{5} \mathrm{G}\right)$.

the forward shock can be significant smaller. In order to detect cosmological GRBs, consequently, it should be desirable larger neutrino detectors, which are impractical, or we should rely on favorable observational conditions that might allow a natural magnification of the signal. One such an effect is gravitational lensing.

\section{Gravitational Lensing of Neutrino Signals from Collapsars}

Neutrinos have zero or negligible mass, so they follow in very good approximation null geodesics. ${ }^{13}$ We consider a point source of neutrinos, with angular diameter distance $D_{\text {os }}$ to the observer, behind a Schwarzschild black hole lens, placed at an angular diameter distance $D_{\text {os }}$. The angular diameter distance between the lens and the source is $D_{\mathrm{ls}}$. The optical axis is defined by the line that joins the observer with the deflector. The distances are very large compared to the Schwarzschild radius of the black hole and the angles are measured from the observer. When the alignment is high, the angular position of the source $\beta$, taken positive here, is small. For this configuration, we have two weak deflection images and two infinite sets of strong deflection (also called relativistic ${ }^{14}$ ) images. Neutrinos with impact parameter $r_{0}$ much larger than the photon sphere radius $r_{\mathrm{ps}}=3 M G / \mathrm{c}^{2}$, will have a small deflection angle $\alpha$, which can be approximated to first order in $1 / r_{0}$ by $\alpha=$ $4 G M /\left(c^{2} r_{0}\right)$ (weak deflection limit). Within this approximation, the lens equation has the form, ${ }^{15}$

$$
\beta=\theta-\frac{\theta_{\mathrm{E}}^{2}}{\theta},
$$


where $\theta$ is the angular position of the image and $\theta_{\mathrm{E}}$ is the angular Einstein radius, given by

$$
\theta_{\mathrm{E}}=\sqrt{\frac{2 R_{\mathrm{s}} D_{\mathrm{ls}}}{D_{\mathrm{ol}} D_{\mathrm{os}}}}
$$

with $R_{\mathrm{s}}=2 M G / c^{2}$ the Schwarzschild radius of the lens. The lens equation has two solutions:

$$
\theta_{\mathrm{p}, \mathrm{s}}=\frac{1}{2}\left(\beta \pm \sqrt{\beta^{2}+4 \theta_{\mathrm{E}}^{2}}\right),
$$

that give the positions of the primary (upper sign) and the secondary (lower sign) images. The primary image lies inside the Einstein radius and the secondary image outside. When $\beta=0$, instead of two images, an Einstein ring with radius $\theta_{\mathrm{E}}$ is obtained. Another important aspect is the magnification of the images, defined as the ratio between the observed and intrinsic fluxes of the source. As a consequence of the Liouville theorem in curved spacetimes, gravitational lensing preserves surface brightness for neutrinos and photons, so the magnifications of the images are given by the ratio of the solid angles subtended by the images and the source, which result in: ${ }^{15}$

$$
\mu_{\mathrm{p}, \mathrm{s}}=\frac{1}{4}\left(\frac{\beta}{\sqrt{\beta^{2}+4 \theta_{\mathrm{E}}^{2}}}+\frac{\sqrt{\beta^{2}+4 \theta_{\mathrm{E}}^{2}}}{\beta} \pm 2\right),
$$

where the plus sign corresponds to the primary image and the minus sign to the secondary one. If the position of the source $\beta$ is close to zero, the magnifications of both images are large. If $\beta=0$ the approximation of point source breaks down and the magnifications become infinite. It is not difficult to see that $\mu_{\mathrm{p}}>1$ for all $\beta$, and $\mu_{\mathrm{s}}>1$ only if

$$
\frac{\beta}{\theta_{\mathrm{E}}}<\sqrt{\frac{(3 \sqrt{2}-4)}{2}} \approx 0.35 .
$$

When $\beta / \theta_{\mathrm{E}}$ is large we have that $\mu_{\mathrm{p}} \approx 1$ and $\mu_{\mathrm{s}} \approx 0$.

The contribution from the relativistic images is in general quite small and can be safely neglected. ${ }^{13}$

Neutrinos that form distinct images take different paths, resulting in time delays between the images. Considering again that neutrinos follow null geodesics as photons do, the time delay between the primary and the secondary images is given by: ${ }^{15}$

$$
\Delta t_{\mathrm{p}, \mathrm{s}}=\frac{2 R_{\mathrm{s}}}{c}\left(1+z_{d}\right)\left(\frac{\theta_{\mathrm{s}}^{2}-\theta_{\mathrm{p}}^{2}}{2\left|\theta_{\mathrm{p}} \theta_{\mathrm{s}}\right|}+\ln \left|\frac{\theta_{\mathrm{s}}}{\theta_{\mathrm{p}}}\right|\right)
$$

where $z_{d}$ is the redshift of the deflector. The last equation can be written in the form

$$
\Delta t_{\mathrm{p}, \mathrm{s}}=\frac{2 R_{\mathrm{s}}}{c}\left(1+z_{d}\right)\left(\frac{-\beta \sqrt{\beta^{2}+4 \theta_{\mathrm{E}}^{2}}}{2 \theta_{\mathrm{E}}^{2}}+\ln \left|\frac{\beta-\sqrt{\beta^{2}+4 \theta_{\mathrm{E}}^{2}}}{\beta+\sqrt{\beta^{2}+4 \theta_{\mathrm{E}}^{2}}}\right|\right) .
$$


When $\beta=0$ there is no time delay. Large time delays can be obtained if $\beta / \theta_{\mathrm{E}} \gg 1$, but in this case the magnification of the primary image is close to one and the secondary image is very faint. The optimal situation for a variable source is when $\beta / \theta_{\mathrm{E}}$ is small enough to have large magnifications of both images, but not too close to zero, so the time delay can be longer than the typical time scale of the transient source.

\section{Results: An Example}

Let us now consider a supermassive black hole at the center of a nearby galaxy. The proximity of the galaxy will result in a rather short time delay, which is highly desirable for detection purposes. A galaxy of such a kind is NGC4486 (M87), with a central black hole of $3.3 \times 10^{9} M_{\odot}$ located at a distance of $15.3 \mathrm{Mpc}$ in the center of Virgo cluster. ${ }^{16}$ For such a black hole the Einstein angle is $\theta_{\mathrm{E}}=1.3$ arcseconds. Assuming an angular position of a background GRB of $\beta=0.1 \theta_{\mathrm{E}}$, we get positions of the primary and secondary images given by $\theta_{\mathrm{p}}=1.05 \theta_{\mathrm{E}}$ and $\theta_{\mathrm{s}}=-0.95 \theta_{\mathrm{E}}$, respectively. The corresponding magnifications are: $\mu_{\mathrm{p}}=5.5$ and $\mu_{\mathrm{p}}=5.5$ and $\mu_{\mathrm{s}}=4.5$. This means an increase in the total number of neutrinos of an order of magnitude respect to what would be expected in absence of gravitational effects. In addition, these neutrinos arrive in two groups, separated by a time lag of $1.3 \times 10^{4} \mathrm{~s}$. This later signature would make the detection secure, and would allow to determine the distance to the GRB (here assumed to be $10^{28} \mathrm{~cm}$ ).

\section{Closing Remarks}

Gravitational lensing, as shown by the previous example, is an important natural tool for the detection of distance transient events generating neutrinos. If the events are Population III GRBs, the detection and measurement of the neutrino signal would be very useful for cosmology and theory of structure formation since such neutrinos were originated in the collapse of the first generation of stars. We can learn from them on the physical conditions at the dawn of the structured universe.

\section{Acknowledgments}

This research was supported by the Argentine Agencies CONICET and ANPCyT through grants PIP 0078 and PICT-2007-00848 BID 1728/OC-AR and by the Ministerio de Educación y Ciencia (Spain) under grant AYA2010- 21782-C03-01, FEDER funds. We thank Ernesto Eiroa for discussions on lensing and Peter Mészáros for valuable comments on neutrinos and GRBs.

\section{References}

1. T. Piran, Rev. Mod. Phys. 76, 1143 (2005).

2. M. Topinka, Balt. Astron. 18, 271 (2009). 
3. S. Woosley, ApJ 405, 273 (1993).

4. P. Mészáros and E. Waxman, Phys. Rev. Lett. 87, 171102 (2001).

5. C. D. Dermer and A. Atoyan, Phys. Rev. Lett. 91, 071102 (2003).

6. A. Tchekhovskoy, J. C. McKinney and R. Narayan, Month. Not. Royal. Astron. Soc. 388, 551 (2008).

7. A. Tchekhovskoy, J. C. McKinney and R. Narayan, ApJ 699, 1789 (2009).

8. S. S. Komissarov, N. Vlahakis, A. Koenigl and M. V. Barkov, Month. Not. Royal. Astron. Soc. 394, 1182 (2009).

9. P. Mészáros and M .J. Rees, ApJ Lett. 733, L40 (2011).

10. S. Gao, K. Toma and P. Mészáros, Phys. Rev. D 83, 103004 (2011).

11. G. E. Romero, F. L. Vieyro and G. S. Vila, $A \mathscr{E} A$ 519, 109 (2010).

12. M. M. Reynoso and G. E. Romero, $A \& A$ 493, 1 (2009).

13. E. Eiroa and G. E. Romero, Phys. Lett. B 663, 377 (2008).

14. K. S. Virbhadra and G. F. R. Ellis, Phys. Rev. D 62, 084003 (2000).

15. P. Schneider, J. Ehlers and E. E. Falco, Gravitational Lenses (Springer-Verlag, Berlin, 1992).

16. K. Gebhardt and J. Thomas, ApJ 700, 1690 (2009). 